\title{
CARACTERÍSTICAS BIOLÓGICAS Y ESPECTRO TRÓFICO DURANTE EL OTOÑO DE DOS POBLACIONES SIMPÁTRICAS DE Blennius fluviatilis Y Micropterus salmoides EN UN EMBALSE PEQUEÑO
}

\author{
José Prenda'* y Emiliano Mellado ${ }^{1 * *}$ \\ 1. Departamento de Biología Vegetal y Ecología, Universidad de Sevilla, Apdo. 1095, 41080-SEVILLA. \\ × Dirección actual: Institute of Freshwater Ecology, River Laboratory, East Stoke, Wareham, Dorset BH20 6BB, REINO UNIDO. \\ ** Dirección de contacto.
}

Palabras clave: peces, España, distribución de tallas, relación longitud-peso, hábitos alimenticios, depredación Keywords: fish. Spain, size distribution, length-weight rclationship, food habits, predation.

\author{
ABSTRACT \\ BIOLOGICAL CHARACTERISTICS AND TROPHIC NICHE DURING AUTUMN IN TWO SYMPATRIC \\ POPULATIONS OF Blennius fluviatilis AND Micropterus salmoides INHABITING A SMALL RESERVOIR
}

Therc exist strong differences in size frequency distribution, feeding habits and in habitat use between the populations of Blennius fluviatilis and Micropterus salmoides coexisting in a small reservoir in NE Spain. Blcnnies are short-sizcd than largemouth bass, its diet is based upon benthic invertebrates, mainly chironomids larvae and inayfly nimphs, and use a habitat without vegetation, madc of large bedrocks and situated in a precise point in the study site. Largemouth bass, on the contrary, use mainly the rcservoir vegetated edges -from which exclude the rest of the fish spccics-, feeds on water coluinn invertebrates (chironomids pupae and zigopterans ninfae) and fishes and are much larger than blennies. These facts, togethcr with a similar behavior of blennies in a ncar site, which lacks largemouth bass (Matarraña river), make us to suppose the Iack of strong interactions between both species, in the sense that one of them were displaced from his optimun habitat. Nonethcless, the presence oî' young largcmouth bass in blennies habitat may be the cause of an inversion in the feeding microhabitat of thc last species.

\section{INTRODUCCIÓN}

Entre los elementos básicos a conocer para aproximarnos a la ccología de los peces, se hallan la descripción de sus principales características poblacionalcs (abundancia, distribución de frecuencias de las tallas, biometría, ctc...) y de su alimentación. En este último caso, el análisis de la dicta de los peces es el punto de partida para el conocimiento de sus requerimientos tróficos, y dc forma indirecta nos permite aproximarnos a sus hábitos de adquisición del alimento, a las interacciones que potencialmente puede mantener con otros individuos (competencia, depredación) e incluso a los patrones de ocupación del espacio.

El blenio de río (Blennius fluviatilis) es una especie de amplia distribución en las aguas epicontinentales de los paises circunmediterráneos. En España se ha citado en las cuencas de los ríos Fluviá, Ebro, Turia, Júcar, Guadiana y Guadalquivir (DOADRIO et al., 1991), aunque existen escasas referencias recientes (VIÑOLAS, 1986; FREEMAN et al., 1990). En España se encuentra en rápida regresión, según DOADRIO et al. (1991) debido a la perdida de calidad de las aguas, a la introducción de especies exóticas y a la destrucción de las zonas de puesta. Ello hace que esté considerada como una especie en peligro de extinción (ICONA, 1986).

El delicado estatus y el desconocimiento que existe del blenio en España hacc particularmente interesante la descripción de las características ecológicas de una de sus poblaciones, que además coexiste con una población de black bass (Micropterus salmoides). Esta última es una especie exótica en las aguas epi- 
continentales europeas, que por su carácter de voraz depredador territorial (SANCHEZ-ISARRIA et al., 1989), ha tenido severas repercusiones sobre las poblaciones de algunos ciprínidos ibéricos (RODRÍGUEZ-JIMÉNEZ, 1989; ELVIRA, 1992).

Teniendo en cuenta estos hechos, en este trabajo se plantean los siguientes objetivos:

a) descripción de algunos parámetros biológicos y poblacionales de las poblaciones de blenio y black bass que coexisten en un embalse de pequeño volumen.

b) descripción de la dieta otoñal de las especies anteriormente mencionadas.

c) a la vista de los resultados obtenidos tratar de lograr una aproximación a las interacciones potenciales existentes entre el blenio y el black bass, especie reconocida como perjudicial para la ictiofauna ibérica autóctona.

\section{ÁREA DE ESTUDIO}

Este trabajo se ha llevado a cabo en la Estanca de Alcañiz (Teruel, NE España). Este es un pequeño embalse de origen romano, destinado en la actualidad a riego con aportaciones de agua procedentes del río Guadalope. Originalmente fué una laguna permanente endorreica. El sustrato sobre el que se asienta es de tipo detrítico con intercalaciones calizas y yesos de la formación de Caspe (ALONSO Y COMELLES, 1985; M.O.P.U., 1986).

TABLA 1. Características físico-quíinicas medias de la Estanca de Alcañiz. Verano de 1991.

TABLE 1. Mean physical and chemical characteristics of the Estanca de Alcañiz. Summer 1991

\begin{tabular}{|c|c|c|}
\hline Temperatura: & 14.0 & ${ }^{\circ} \mathrm{C}$ \\
\hline $\mathrm{pH}:$ & 8.1 & \\
\hline Conductividad: & 606.5 & $\mu \mathrm{Scm}^{\prime}$ \\
\hline Oxígeno disuelto: & 9.7 & $\operatorname{mg} 021^{1}$ \\
\hline Secchi: & 1.9 & $\mathrm{~m}$ \\
\hline Residuo seco: & 3.1 & $\mathrm{mg} \mathrm{l}^{-1}$ \\
\hline O.Q.D.: & 14.3 & $\operatorname{mg~} \mathrm{O}_{2} \mathrm{l}^{1}$ \\
\hline Alcalinidad: & 373.0 & $\mathrm{mg} \mathrm{CaCO}{ }^{1}$ \\
\hline Dureza total: & 347.5 & $m g \mathrm{CaCO}_{3} 1^{1}$ \\
\hline N-NO,-: & 12.5 & $\mathrm{mg} \mathrm{l}^{-1}$ \\
\hline N-N02: & 1.0 & $\mu g$-at $\mathrm{N}^{1}$ \\
\hline N-NH-4: & 0.6 & $m g \mathrm{I}^{\mathrm{s}}$ \\
\hline Fósforo total: & 0.9 & pg-at $\mathrm{Pl}^{\mathbf{1}}$ \\
\hline Clorofila: & 1.9 & $\mathrm{~g}^{-1}$ \\
\hline Volumen: & 6.9 & hrn' \\
\hline Superficie: & 151 & ha \\
\hline Perímetro: & 5 & $\mathrm{~km}$ \\
\hline Longitud máxima: & 1.5 & $\mathrm{~km}$ \\
\hline Anchura máxima: & 1 & $\mathrm{~km}$ \\
\hline Profundidad media: & 4.5 & $\mathrm{~m}$ \\
\hline Profundidad máxima: & 14 & $\mathrm{~m}$ \\
\hline
\end{tabular}

Las principales características morfométricas y limnológicas de la Estanca se recogen en la Tabla 1. Las aguas son moderadamente mineralizadas y su contenido en nutrientes tambien moderado. Durante la realización del estudio, la temperatura fué de $8,6^{\circ} \mathrm{C}$, el pH de 8,3 , la conductividad de $650 \mu \mathrm{S} \mathrm{cm}{ }^{3}$ y la concentración de oxígeno disuelto osciló entre 7 y $9 \mathrm{mg}^{1}$. La turbiedad del agua era apreciable, la vegetación de orillas escasa, compuesta por manchas laxas de Scirpus sp. y de Phragmites communis. En una zona somera próxima a la entrada del canal procedente del río Guadalope se localizó una superficie importante cubierta del macrófito sumergido Potamogeton sp.

La ictiofauna de la Estanca de Alcañiz está compuesta por 10 especies, algunas de ellas autóctonas, pero la mayoría han sido introducidas por el hombre en épocas recientes. En la Tabla 2 se presenta una lista con la composición cualitativa de la ictiofauna de la Estanca durante la época en que se llevó a cabo el muestreo. Las aguas libres están dominadas por madrillas (Chondrostoma toxostoma) y carpas comunes (Cyprinus carpio), mientras que en orillas la especie más abundante es el black bass.

TABLA 2. Ictiofauna presente en la Estanca de Alcañiz.

TABLE 2. Fish species captured in the Estanca de Alcañiz during November 1991 and other species cited before this date.

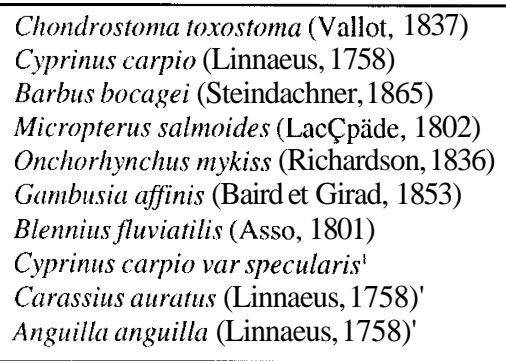

\section{MATERIAL Y MÉTODOS}

El muestreo se llevó a cabo en Noviembre de 1991. Para la captura de los peces se emplearon redes holandesas y pesca eléctrica, ambos métodos en zonas someras con profundidades inferiores a $1.5 \mathrm{~m}$. A lo largo del perímetro de la Estanca se establecieron 12 puntos de muestreo homogéneamente distribuidos, en 10 de los cuales se colocaron tres nasas de cuatro muertes cada una. Cada grupo de tres nasas colocado durante 24 horas constituyó una unidad de esfuerzo. En cada punto de muestreo se hizo una estima visual del porcentaje de cobertura de la vegetación emergente.

Las muestras de blenio se tomaron con pesca eléctrica (220 $\mathrm{V}, 2-5 \mathrm{~A}$ ) en la zona de influencia del canal de alimentación de la Estanca. Por cada ejemplar capturado se registró in situ su 
longitud total en milimetros (LT) y peso fresco total en gramos (PT). Los individuos fueron etiquetados y conservados en formol al $4 \%$ para su transporte, y posterior análisis en laboratorio.

Para el estudio de la dieta, los estómagos junto con el resto del tracto digestivo fueron separados del ejemplar y conservados en formaldehido al $4 \%$. El contenido fué vaciado y observado bajo lupa binocular $(\mathrm{x} 10, \mathrm{x} 40)$ y microscopio óptico (x100, $\mathrm{x} 400$ ). Las presas se determinaron al nivel taxonómico más bajo posible. Para la evaluación cuantitativa de la dieta se determinó, en primer lugar, el porcentaje de llenado del tracto digestivo. En segundo lugar, se registró la abundancia (A) de cada presa en el total del contenido alimenticio, como el porcentaje respecto al

\section{a) Blennius fluviatilis}

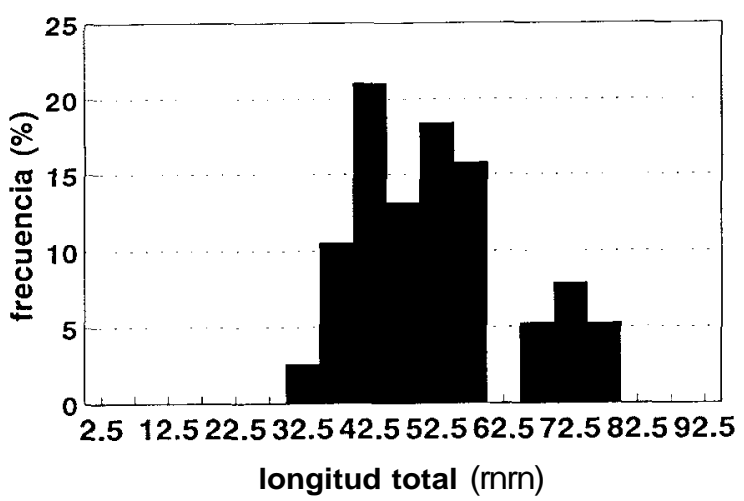

b) Mícropferus salmoides

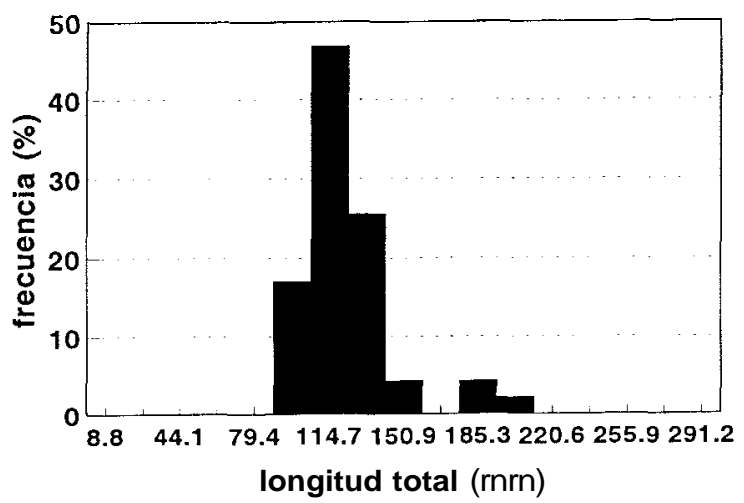

FIGURA 1. Distribución de frecuencias de las tallas de Blennius fluviatilis y Micropterus salmoides capturados en la Estanca de Alcañiz (Teruel, España) en Noviembre de 1991

FIGURE 1. Size frequency distribution of Blennius fluviatilis and Micropterus salmoides captured in Estanca de Alcañiz reservoir (Teruel, España) in November 1991 número total de individuos contabilizados y la ocurrencia $(\mathrm{O})$ de cada presa como el número de estómagos con dicha categoría alimenticia del total de estómagos analizados.

Para identificar los especimenes y estimar el peso y la talla de los peces depredados se emplearon las claves de identificación y las rectas de regresión entre la longitud de los huesos encontrados en los estómagos y la longitud estandar y el peso fresco total de los peces, según las claves y las ecuaciones elaboradas por PRENDA y GRANADO-LORENCIO (1992, a, b).

Se valoró la amplitud de nicho trófico con los índices $\mathrm{H}^{\prime}$ de Levins (LEVINS, 1968) y B de Simpson (en RACHLIN et al., 1989). Para cuantificar el solapamiento trófico intraespecífico e interespecífico se utilizaron los índices Á' de Pianka (PIANKA, 1973) y Á' de Schoener (en RACHLIN et al., 1989).

\section{RESULTADOS}

\section{Características biológicas}

\section{Blennius fluviatilis}

En la zona del canal de entrada de la Estanca se recolectaron 52 ejemplares de blenio. Aparte de esta especie se capturaron 3 ejemplares de black-bass, 1 de carpa común y 1 de gambusia.

La distribución de frecuencias de las tallas del blenio presenta tres picos (Fig. la). Esta distribución es similar a la obtenida por VIÑNOLAS (1986) para la misma especie en el vecino río Matarraña. Esta autora establece tres clases de edad, que pueden coincidir con las de la población de la Estanca: una clase $0+$ con tallas en torno a los $40 \mathrm{~mm}$, una $1+$ con longitudes alrededor de $50 \mathrm{~mm}$ y una tercera clase $2+$, con tallas cercanas a los $70 \mathrm{~mm}$ (esta es claramente superior a la del Matarraña).

Las ecuaciones de regresión de la longitud total y el peso fresco total aparecen en la Tabla 3.

TABLA 3. Relación longitud-peso según la ecuación $\mathrm{W}=\mathrm{aL}^{\mathrm{\vdash}}$ ( $\mathrm{W}=$ peso total, $\mathrm{L}=$ longitud total) para el blenio (Blennius fluviatilis) y para el black bass (Micropterus salmoides) capturados en la Estanca de Alcañiz en Noviembre de 1991.

TABLE 3. Length-weight relationship expressed by the equation $W=a L^{n}(W=$ total weight, $\mathbf{L}=$ total lenght) for blennies (Blennius fluviatilis) and largemouth bass (Micropterus salmoides) captured in the Estanca de Alcañiz in November 1991.

\begin{tabular}{lccccc}
\hline Especie & Loga & $\mathbf{b}$ & $\mathbf{R}^{2}$ & $\mathbf{F}$ & $\mathbf{P}$ \\
\cline { 1 - 5 } Micropterus salmoides & -10.72 & 2.88 & 92.3 & 542.7 & 0.00 \\
Blennius fluviatilis & -12.89 & 3.35 & 98.9 & 1586.8 & 0.00 \\
\hline $\mathrm{a}=$ ordenada en el origen & & & & & \\
$\mathrm{b}=$ pendiente & & & & & \\
$\mathrm{R}^{2}=$ cocficiente de corrclación & & & & \\
\hline
\end{tabular}




\section{Micropterus salmoides}

Aparte de los 3 ejemplares de black bass capturados con pesca eléctrica, el resto (39) se capturó con redes holandesas. El black bass con más del $85 \%$ de las capturas fué la especie más abundante en los hábitats de orillas de la Estanca, apareciendo como única especie en la mayoría de los puntos de muestreo, exceptuando dos puntos que aparecieron vacíos y otro que solo contenía una carpa y un ejemplar de trucha arco-iris.

La distribución de frecuencias de las longitudes del black bass aparecen en la Fig. 1b. En ella se pone de manifiesto la existencia de tres probables clases de edad. Una con tallas inferiores a $80 \mathrm{~mm}$, otra con tallas comprendidas entre $80 \mathrm{y}$ $170 \mathrm{~mm}$, con una moda de unos $110 \mathrm{~mm}$ y otra con longitudes mayores de $170 \mathrm{~mm}$.

\section{Alimentación \\ Blennius fluviatilis}

En primer lugar se debe destacar la dieta exclusivamente carnívora de esta especie (Tabla 4). No se ha encontrado ningún tipo de resto vegetal, ni de detritos en los tractos digestivos analizados. En ellos se han determinado un total de 10 taxa, 9 correspondientes a artrópodos y uno a peces. De los artrópodos uno es de origen terrestre (ortóptero), mientras que los ocho restantes son acuáticos.

Las presas fueron en todos los casos de pequeño tamaño, dominando las ninfas de efemerópteros $(A=57.9 \%, O=75 \%)$ (Tabla 4). Entre éstas se logró determinar la presencia de ejemplares pertenecientes a la familia Leptophlebidue, y dentro de ésta a algunos pertenecientes al género Thraulus. En ninguno de estos casos ha sido posible cuantificar con precisión el número de individuos pertenecientes a ambos taxa, por lo que han sido asignados al nivel taxonómico de los efemerópteros. La segunda presa en importancia la constituyen las larvas de quironómidos ( $\mathrm{A}=38.5 \%, \mathrm{O}=40 \%$ ), la mayoría pertenecientes a la subfamilia Orthocladinae, de hábitos reófilos. La presencia de restos de peces en dos casos es un interesante dato que pone de manifiesto su ictiofagia y voracidad, a pesar de su pequeño tamaño. En el mismo periodo, la dieta del blenio en el río Matarraña (VIÑOLAS, 1986) fué muy similar, aunque dominaron los quironómidos $(\mathrm{A}=56.2 \%)$ frente a los efemerópteros $(\mathrm{A}=16.2 \%)$.

Para evaluar los posibles cambios en la composición de la dieta con la talla, los ejemplares analizados se agruparon en tres categorias de tamaño, según las clases de edad obtenidas por VIÑOLAS (1986) en el río Matarraña. Esto se consiguió
TABLA 4. Composición de la dieta de los blenios (Blennius fluviatilis) y black bass (Micropterus salmoides) en la Estanca de Alcañiz en Noviembre 1991. Los datos se expresan como abundancia (frecuencia de aparición de un determinado item respecto al total de las presas contabilizadas) y como ocurrencia (frecuencia de aparición de un determinado item en el conjunto de los estómagos analizados) $\mathrm{N}=\operatorname{ninfa}, \mathrm{P}=$ pupa, $\mathrm{L}=$ larva, $\mathrm{n}=$ número de ejemplares analizado. $\mathrm{A}=$ abundancia, $\mathrm{O}=$ ocurrencia.

TABLE 4. Diet composition of blennies (Blennius fluviatilis) and largemouth bass (Micropterus salmoides) from the Estanca de Alcañiz in November 1991. The data are expressed as abundance (frequency of each item with respect to all the preys counted) and as occurrence (frequency of each item with respect to the stomach analyzed). $\mathrm{N}=$ nymph, $\mathrm{P}=$ pupae, $\mathrm{L}=$ larvae, $\mathrm{n}=$ number of fish sampled. $\mathrm{A}=$ abundance, $\mathrm{O}=$ occurrence.

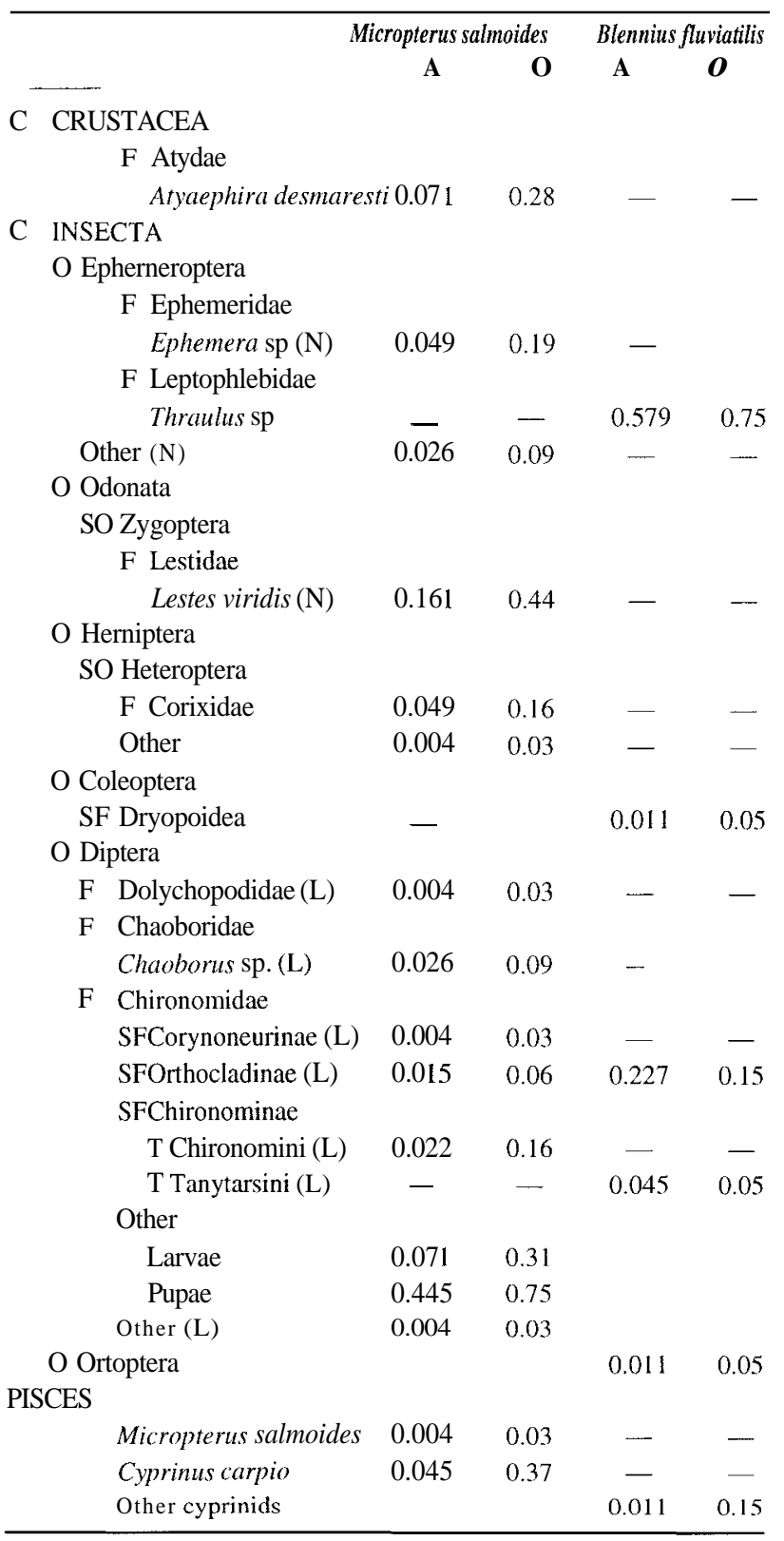


agrupando a los individuos con longitud total inferior a $45 \mathrm{~mm}$ (clase de edad $0+$ ), a los comprendidos entre 45-60 mm (clases de edad $1+$ y $2+$ ) y a los mayores de $60 \mathrm{~mm}$ (clase de edad $3+$ ), y se representó la frecuencia de aparición de los diversos items para cada una de estos grupos (Fig. 2a). Los resultados muestran que la dieta es independiente de la talla (Tabla de contingencia $3 * 7, \mathrm{G}=-3.73,4$ g.l., $\mathrm{P}=0.001$ ). A pesar de ello, se observa una cierta tendencia hacia un incremento en el consumo de efcmerópteros con la talla, en detrimento del consumo de larvas de quironómidos, justo al contrario de lo observado por VIÑOLAS (1986), para un ciclo anual. Según esta misma autora esta tendencia se debe a un cambio en el microhábitat de alimentación, los ejemplares menores tienden a alimentarse prefcrencialmente $\mathrm{cn}$ las orillas someras del río (donde dominan las ninfas de efemerópteros, principalmente de leptoflébidos), y conforme avanza la edad la especie tiende a abandonar las zonas marginales para ocupar las más profundas (donde son dominantes los quironómidos).

La amplitud de nicho trófico, medida con el índice de Levins o con el de Simpson (Tabla 5) es rclativamcrite estrecha para esta especie. Por tallas, se observa un dccremento en la diversidad de presas consumidas conforme se hacen mayores los blenios. Aunque hay que tener en cuenta que la muestra de los individuos de mayor talla es de solo 3 ejemplares. El solapamiento trófico de los tres grupos de tallas cs muy elevado, tal como era de esperar, siendo mayor en los grupos adyacentes que en los extremos. En el río Matarraña la amplitud trófica, medida con el índice de Levins, es muy superior (VIÑOLAS, 1986).

\section{Micropterus salmoides}

Al igual que lo ocurrido con el blenio, el black bass presenta una dieta completamente carnívora (Tabla 4). En total se han identificado 15 taxa en los contenidos estomacales analizados. Dc ellos uno es un crustáceo, 13 son insectos y dos peces. Desde un punto dc vista cualitativo, la dieta de esta especie, en la época de estudio, está basada en invertebrados acuáticos. Desde un punto de vista cuantitativo el item más consumido es el coristituido por las pupas de quironómidos $(A=44.5 \%$, $\mathrm{O}=75 \%$ ), seguido de las ninfas del zigóptero Lestes viridis $(\mathrm{A}=16.1 \%, \mathrm{O}=44 \%$ ) (Tabla 4 ).

La ictiofagia del blac kbass queda de manifiesto por el consumo de individuos de carpa (C. carpio) y de uno de su propia cspecic. Este canibalismo puede ser indicativo de situaciones poco favorables cn las que sc ven obligados a depredar sobre conespecíficos. Esta hipótesis se vc apoyada por el bajo porcentaje de llenado, tanto del estómago (media $=26.5 \%, \mathrm{sd}=32.2$ )
'TABLA 5 Amplitud de nicho trófico, medido con los índices H' de Levin y B de Simpson para Blennius fluviatilis, y black bass considerado en conjunto, y para las tres clases de tallas en que se ha dividido la muestra, y grado de solapamiento trófico entre ellas, medido con los índices de Pianka y Schoener. Para comparar se incluyen datos del embalse de Proserpina (Rodríguez-Jiménez, 1989) y del río Matarraña (VINOLAS, 1986).

TABLE 5. Trophic niche width of Blennius fluviatilis and Micropterus salmoides samples, measured with Levin's H' and Simpson's B, both considering each sample as a whole and for each one of the three size classes in which the sample has been divided. Also the trophic overlapping between the three size classes, measured with Pianka and Schoener indexes, is shown. To compare. some data of Proserpina reservoir (Rodríguez-Jiménez, 1989) and Matarraña river (VINOLAS, 1986) are included.

\begin{tabular}{|c|c|c|c|}
\hline \multicolumn{4}{|c|}{ AMPLITUD DE NICHO TROFICO } \\
\hline & $\mathrm{n}$ & $\mathrm{H}^{\prime}$ & $\mathrm{B}$ \\
\hline \multicolumn{4}{|l|}{ Blennius fluviatilis } \\
\hline muestra conipleta: & 20 & 1.14 & 0.42 \\
\hline < 45 mm (Grupo A): & 9 & 1.12 & 0.50 \\
\hline 45-60 mm (Grupo B): & 8 & 0.97 & 0.38 \\
\hline$>60$ mm (GrupoC): & 3 & 0.76 & 0.33 \\
\hline \multicolumn{4}{|l|}{ RÍO MATARRAÑA } \\
\hline riiucstra coinpleta: & 26 & 2.04 & 0.25 \\
\hline$<45 \mathrm{~mm}(\mathrm{~A})$ & 46 & 1.73 & 0.20 \\
\hline $45-53 \operatorname{inin}(R)$ & 48 & 0.83 & 0.09 \\
\hline $54-61 \mathrm{~mm}(\mathrm{C})$ & 42 & 1.04 & 0.10 \\
\hline$>61 \mathrm{~mm}(\mathrm{D})$ & 28 & 1.51 & 0.19 \\
\hline \multicolumn{4}{|l|}{ Micropterus salmoides } \\
\hline muestra completa: & 33 & 2.47 & 0.43 \\
\hline$<110 \mathrm{~mm}($ Grupo A): & 11 & 2.35 & 0.48 \\
\hline 110-145 mm (Grupo B): & 20 & 2.41 & 0.38 \\
\hline$>145$ nirn (Grupo C): & 2 & 2.13 & 0.49 \\
\hline \multicolumn{4}{|l|}{ EMBALSE DE PROSERPINA } \\
\hline niuestra completa: & 86 & 2.13 & 0.31 \\
\hline 100-180 (primavera): & 25 & 1.90 & 0.24 \\
\hline 100-180 (verano): & 31 & 2.51 & 0.32 \\
\hline < 100 mm (verano): & 30 & 1.97 & 0.37 \\
\hline \multicolumn{4}{|c|}{ SOLAPAMIENTO TRÓFICO } \\
\hline & \multicolumn{2}{|c|}{ Pianka } & Schocner \\
\hline \multicolumn{4}{|l|}{ Blennius fluviatilis } \\
\hline Grupo A-Grupo B: & \multicolumn{2}{|c|}{0.91} & 77.12 \\
\hline Grupo A-Grupo C: & \multicolumn{2}{|c|}{0.58} & 42.18 \\
\hline Grupo B-Griipo C: & \multicolumn{2}{|c|}{0.86} & 65.06 \\
\hline Grupo A-Grupo B-Grupo C: & \multicolumn{2}{|c|}{0.77} & \\
\hline \multicolumn{4}{|l|}{ Microptcrus salmoides } \\
\hline Gi-tipo A-Cr-upo B: & \multicolumn{2}{|c|}{0.87} & 69.82 \\
\hline Grupo A-Grupo C: & \multicolumn{2}{|c|}{0.87} & 69.51 \\
\hline Grupo B-Grupo C: & \multicolumn{2}{|c|}{0.95} & 81.11 \\
\hline Grupo A-Grupo B-Grupo C: & \multicolumn{2}{|c|}{0.90} & \\
\hline \multicolumn{4}{|l|}{ PROSERPINA } \\
\hline $100-180 /<100:$ & & & \\
\hline
\end{tabular}


como del tracto digestivo completo (media $=21.5 \%$, $\mathrm{sd}=17.1$ ). No obstante, este canibalismo ha sido reportado por otros autores (TOMELLERI \& EBERLE, 1990; GÓMEZ CARUANA y DÍAZ LUNA, 1991). La talla media de las carpas consumidas fué de $38.9 \mathrm{~mm}$ (rango=32.5-44.6 mm) correspondiéndose probablemente con individuos nacidos en el año.

\section{a) Blennius fluviatilis}

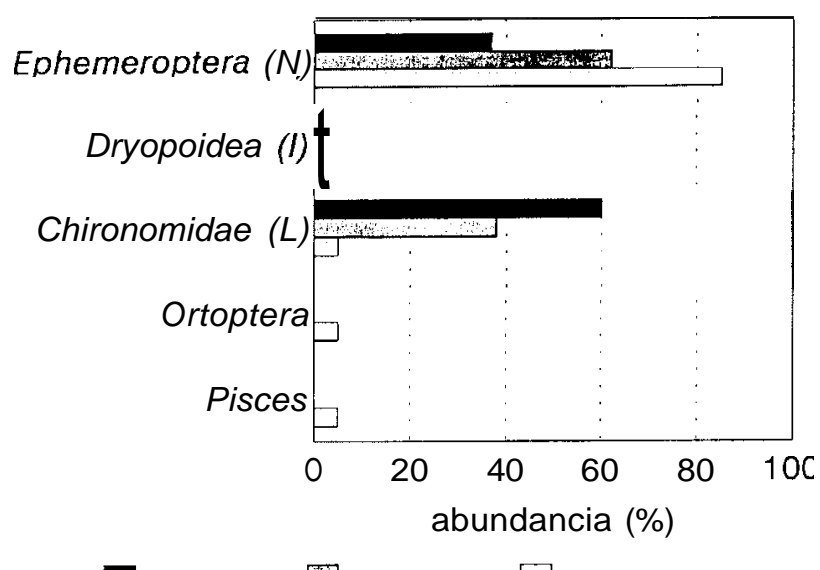

$<45 \mathrm{~mm}$ 图 45-60 mm $\square>60 \mathrm{~mm}$

\section{b) Micropterus salmoides}

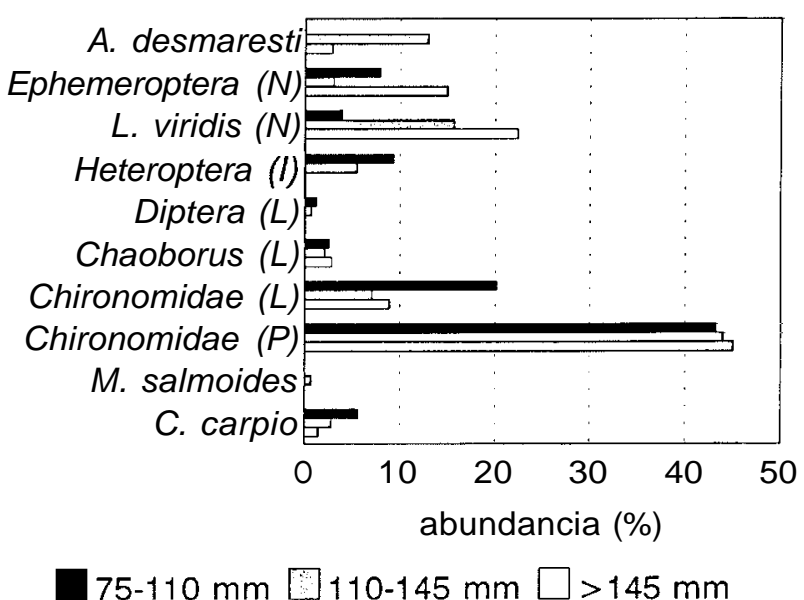

FIGURA 2. Cambios en la dieta con la talla para el blenio (Blennius fluviatilis) y el black bass (Micropterus salmoides) en La Estanca de Alcañiz, Noviembre de 1991, basado en la abundancia (en \%) de cada tipo de presas.

FIGURE 2. Diet shifts with size for freshwater blenny (Blennius fluviatilis) and largemouth bass (Micropterus salmoides) in La Estanca reservoir, November 1991 , based in the abundance (in \%) of each prey item.
La dieta del black bass en la Estanca (Fig. 2b) es dependiente de la talla de los ejemplares (tabla de contingencia de $7 * 3, \mathrm{G}=47.31,12$ g.1., $\mathrm{P}=0.001$ ), coincidiendo con lo observado por SÁNCHEZ-ISARRIA et al. (1989). Es destacable el hecho de que las tallas menores no consumen A. desmaresti, que son depredadas, principalmente, por las intermedias. Las ninfas de L. viridis son presa principal de las tallas intermedias y superiores en detrimento de las menores. Lo contrario ocurre con las larvas de quironómidos y con los alevines de carpa.

La amplitud de nicho trófico de las tres clases de talla de la Estanca es muy similar entre ellas y relativamente superior a las calculadas para la misma especie en un embalse de características similares, situado en la provincia de Badajoz (E. de Proserpina) en primavera, e inferior en verano (Tabla 5) (RODRÍGUEZ JIMÉNEZ, 1989). El solapamiento trófico es muy elevado entre las tres categorias de tallas. Es superior entre los grupos B y C e inferior entre los grupos A y B y A y C. En el caso de Proserpina el solapamiento es muy inferior.

\section{DISCUSIÓN}

\section{Características biológicas}

La zona de muestreo donde se capturaron los blenios se caracterizó por la presencia de rocas de gran tamaño entre las que quedaban pequeñas charcas. La vegetación acuática era muy escasa y la profundidad variable, entre $0.5 \mathrm{~m}$ y algunos centímetros. La presencia de pequeños ejemplares de black bass en la zona, sugiere la posibilidad de que se establezcan interacciones entre éstos y los blenios, bien competitivas, bien de depredación.

A pesar de ello, los ejemplares de black bass fueron mayoritariamente capturados en hábitats someros de orillas, existiendo una alta correlación positiva entre las capturas por unidad de esfuerzo de esta especie y el porcentaje de cobertura vegetal (coeficiente de correlación por rangos de Kendall, Ó=0.7475, $\mathrm{P}=0.001$ )

Se puede concluir que existe una disjunción parcial entre los hábitats utilizados por el blenio y por el black bass. Esta última especie, por su carácter de depredador territorial, puede excluir con bastante eficiencia al resto de las especies de su hábitat óptimo, que se pueden calificar de accidentales en las zonas someras de orillas. Sin embargo, el blenio ocupa en la Estanca un hábitat marginal de características similares al ocupado en el río Matarraña, incluso en su carácter fluctuante (VIÑOLAS, 1986; FREEMAN et al., 1990). 


\section{Alimentación}

Las presas consumidas por el blenio son típicos pobladores del bentos (ninfas de efemerópteros, larvas de quironómidos), de donde se puede deducir que esta especie utiliza poco la columna de agua o la superficie para alimentarse, al igual de lo que ocurre en el río Matarraña (VIÑOLAS, 1986). Ello puede deberse a que el blenio al carecer de vejiga natatoria, está obligado a permanecer en el fondo.

Una característica común a casi todos los invertebrados depredados por el black bass es la de ser más o menos limnéfilos y localizarse en la columna de agua (A. desmaresti, heterópteros, coríxidos, Chaoborus y pupas de quironómidos). Ello nos está indicando un tipo de alimentación, basado casi por completo en la columna de agua. Esto concuerda con los resultados obtenidos por otros autores (ver KEAST, 1985) y que viene determinado en gran medida por sus características anatómicas, en las que destaca su boca terminal súpera, y su estrategia de caza al acecho.

La mayor diversidad trófica del black bass en la Estanca en comparación con el embalse de Proserpina (RODRÍGUEZJIMÉNEZ, 1989) (Tabla 5), puede deberse a una menor abundancia de presas en el primer medio, que obliga a esta especie a diversificar su dieta en él (SCHOENER, 1971). Las escasas diferencias en la amplitud de nicho trófico entre las distintas clases de talla en la Estanca respecto a Proserpina pueden deberse al estrecho rango que presenta cada clase en el primer embalse respecto al segundo.

La estrategia alimenticia seguida por ambas especies es similar. En ambas existen una (black bass) o a lo sumo dos (blenio) presas dominantes (consumidas por un mayor número de ejemplares en mayor cantidad) y el resto de los items se pueden calificar de raros, es decir son consumidos por un número bajo o medio de individuos. pero en muy baja cantidad (COSTELLO, 1990) (Fig. 3).

La dieta del black bass es significativamente diferente de la del blenio (Tabla de contingencia $2 * 11$, chi cuadrado $=158.7$, 10 g.l., $P=0.001$ ). Por otro lado, la diversidad de presas consumidas por el black bass o su amplitud de nicho trófico es marcadamente superior a la del blenio, si la medimos con el índice H' de Levin y similar si consideramos el índice B de Simpson. En cualquier caso, el número de presas distintas consumidas por el black bass es muy superior al del blenio (16 en el primer caso, frente a 8 en el segundo). Ello, sin duda, se debe a su capacidad de explotar, tanto el bentos, como la columna de agua o la superficie de la misma y a su mayor capacidad de movimientos. Esto lo convierte en un potencial competidor del blenio, aunque en el caso que nos ocupa presentan un bajo solapamiento trófico (Á' Pianka=0.25, Á' Schoener $=$ $21.02 \%)$, consumiendo diferentes presas y en diferentes proporciones.

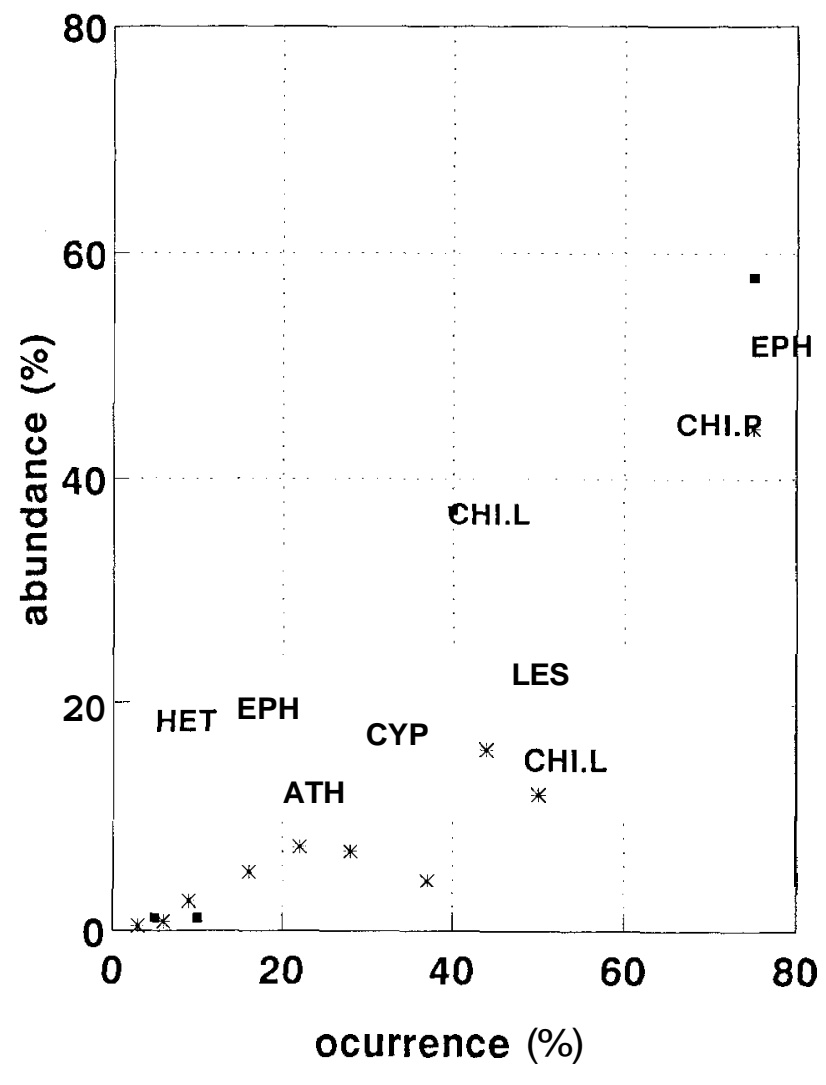

- B. fluviatilis * M. salmoides

FIGURA 3. Representación gráfica de la frecuencia de ocurrencia frente a la abundancia (ambas en \%) de presas en la dieta de los blenios (Blennius fluviatilis) y black bass (Micropterus salmoides) capturados en la Estanca. EPH: ninfas de efemerópteros, CHI.L: larvas de Chironomidae, CHI.P: pupas de Chironomidae, LES: Lestes viridis, CYP: Cyprinus carpio, ATH: Athyaephira desmaresti, HET: heteróptera.

FIGURE 3. Plot of frequency occurrence versus abundance (both in \%) of prey in the diet of fish collected in Estanca reservoir. EPH: ephemeroptera ninfae, CHI.L: chironomidae larvae, CH1.P: chironomidae pupae, LES: Lestes viridis, CYP: Cyprinus carpio, ATH: Athyaephira desmaresti, HET: heteroptera.

En otras zonas con características similares a la Estanca, como el ya citado embalse de Proserpina en Badajoz, el black bass, junto al pez sol (Lepomis gibbosa) y a la gambusia (Gambusia affinis) han eliminado casi por completo a la antigua asociación de alevines, jaramugos y adultos de Rutilus lemmingi, Leuciscus pyrenaicus, Cobitis maroccana, Barbus bocagei y Cyprinus carpio (RODRÍGUEZ-JIMÉNEZ, 1989). Si corisideramos que para determinar la existencia de interaccio- 
nes entre las dos especies que nos ocupan es necesario conocer los hábitos del blenio en una zona en la que no esté presente el black bass (ROSS, 1991) y teniendo en cuenta los resultados obtenidos por VIÑOLAS para la especie en el río Matarraña en el que no existen black bass ni especies similares, se puede hipotetizar que en la Estanca no se registran interacciones fuertes durante el periodo de estudio.

No obstante, si consideramos la existencia de una relación inversa en el consumo de larvas de quironómidos y ninfas de efemerópteros y su relación con el microhábitat de alimentación según propone VIÑOLAS (1986), entre los blenios de la Estanca y los del río Matarraña, se puede plantear la hipótesis de un efecto del black bass. Es decir, la presencia del black bass en la Estanca invertiría la calidad de los microhábitats en el sentido de convertirse en óptimos los de orillas someras, fuera de la acción de los competidores/depredadores (black bass), frente a los de zonas más profundas en los que estarían presentes estos últimos, al igual que lo observado experimentalmente por otros autores (POWER, 1987; SCHLOSSER, 1988).

En resumen, en la Estanca de Alcañiz, tanto black bass, como blenios ocupan un hábitat óptimo, aunque en el caso de estos últimos la presencia de individuos jóvenes de black bass puede ser responsable de una inversión en el sentido "esperable" de la ocupación del mismo (PRENDA \& GRANADOLORENCIO, en prensa), de manera que los "microhábitats óptimos" o más profundos (sensu VIÑOLAS, 1986) son ocupados por los individuos de menor talla, frente a los de orillas que son utilizados por los mayores.

\section{AGRADECIMIENTOS}

Los autores desean agradecer al Servicio de Conservación del Medio Natural de la Diputación General de Aragón las facilidades prestadas para la realización de este trabajo. Igualmente expresan su agradecimiento a Luis Bolea, a José Luis, Presidente de la Sociedad de Pescadores de Alcañiz, a Manolo del Bar La Perca y a la Guarderia Forestal de esta localidad, por su inestimable ayuda y colaboración en las tareas de campo.

La Dra. L. Encina hizo valiosas sugerencias sobre una primera versión del manuscrito.

\section{BIBLIOGRAFÍA}

ALONSO, M. y M. COMELLES. 1985. Catálogo limnológico de las balsas y lagunas de la provincia de Teruel. Teruel, 73:66-75.
COSTELLO, M. J. 1990. Predator feeding strategy and prey importance: a new graphical analysis. Journal of Fish Biology, 36: $261-263$.

ELVIRA, B. 1992. Freshwater fishes introduced in Spain and relationships with autochthonous species. World Fisheries Congress, Atenas.

FREEMAN, M. C., D. VIÑOLAS, G. D. GROSSMAN y A. DE SOSTOA. 1990. Microhabitat use by Blennius fluviatilis in the Rio Matarraña, Spain. Freshwat. Biol, 24: 335-345.

GÓMEZ CARUANA, F. y J. L. DÍAZ LUNA. 1991. Guía de los peces continentales de la Península Ibérica. Ed. Penthalon, Madrid.

ICONA. 1986. Lista Roja de los Vertebrados de España. Pub. del Ministerio de Agricultura, Pesca y Alimentación.

KEAST, A. 1985. The piscivore feeding guild of fishes in small freshwater ecosystems. Environmental Biology of Fishes, 12: 119-129.

LEVINS, R. 1968. Evolution in Changing environments. Princenton University Press, Princenton NJ.

M.O.P.U. 1986. Inventario de presas españolas. Centro de Publicaciones. Secretaria General Técnica, Madrid.

PIANKA, E. R. 1973. The structure of lizard communities. Annu. Rev. Ecol. Syst., 4: 53-74.

POWER, M. 1987. Predator avoidance by grazing fishes in temperate and tropical streams: importance of stream depth and prey size. En Predation. Direct arzd indirect effects in aquatic communities (Eds. W. C. Kerfoot y A. Sih). University Press of New England, Hannover: 331-351.

PRENDA, J. y C. GRANADO-LORENCIO. 1992 a. Biometric analysis of some cyprinid bones to estimate the original length and weight of prey fishes. Folia Zool, 41: 175-183.

PRENDA, J. y C. GRANADO-LORENCIO. 1992 b. Claves de identificación de Barbus bocagei, Chondrostoma polylepis, Leuciscus pyrenaicus y Cyprinus carpio mediante algunas de sus estructuras oseas. Doñana, Act. Vert., 19: (en prensa).

PRENDA, J. y C. GRANADO-LORENCIO. En prensa. Fish microdistribution in a stream in Southern Spain: Predator avoidance or microhabitat preference'? Verh. Internat. Verein. Limnol.

RACHLIN, J. $W$., B. E. WARKENTINE y A. PAPPANTONIOU. 1989. The use of niche breadth and proportional similarity in feeding to stipulate resource utilization strategies in fish. J. Freshwater Ecol, 5: 103-112.

RODRÍGUEZ-JIMÉNEZ, A. J. 1987. Relaciones tróficas de una comunidad íctica, durante el estío en el río Aljucén (Extremadura, España). Misc. Zool., 11: 249-256.

RODRÍGUEZ-JIMÉNEZ, A. J. 1989. Hábitos alimenticios de Micropteus salmoides (Pisces: Centrachidae), Lepomis gib- 
bosus (Pisces: Centrachidae) y Gambusia affinis (Pisces: Poecilidae) en las orillas del embalse de Proserpina (Extremadura, España). Limnética, 5: 13-20.

ROSS, S. T. 1991. Mechanisms structuring stream fish assemblages: are there lessons from introduced species? Environmental Biology of Fishes, 30: 359-368.

SÁNCHEZ-ISARRIA, M. A., L. CABANAS, A. SOSA, E. TUTOR y S. PELLICER. 1989. Análisis de la composición de la dieta en el medio natural del black-bass (Micropterus salmoides, Lcp.) de tamaño mayor de $20 \mathrm{~cm}$. Act. I Col. Luso-Esp. Bacias Hidrogr. e Rec. Zool: : 359-364.
SCHLOSSER, I. J. 1988. Predation risk and habitat selectionby two size classes of a stream cyprinid: experimental test of a hypothesis. Oikos, 52: 36-40.

SCHOENER, T. W. 1971. Theory of feeding strategies. Ann. Rev. Ecol. Syst., 2: 369-404.

TOMELLERI, J. R. y M. E. EBERLE. 1990. Fishes of the Central United States. University Press of Kansas, Kansas.

VIÑOLAS, D. 1986. Biologia i ecologia de Blennius fluviatilis Asso, 1801, en el riu Matarraña. MSc Thesis, University of Barcelona. 